\title{
Single Crystal of a One-dimensional Covalent Organic Framework
}

Hai-Sen Xu,${ }^{1} \dagger$ Yi Luo, ${ }^{2,3} \uparrow$ Xing Li, ${ }^{1} \uparrow$ Pei Zhen See, ${ }^{1}$ Zhongxin Chen, ${ }^{1}$ Tianqiong Ma,${ }^{2}$ Lin Liang, ${ }^{4}$ Kai Leng, ${ }^{1}$ Ibrahim Abdelwahab, ${ }_{1}^{1}$ Lin Wang, ${ }^{1}$ Runlai Li, ${ }^{1}$ Xiangyan Shi, ${ }^{5}$ Yi Zhou, ${ }^{6}$ Xiu Fang Lu, ${ }_{1}$ Xiaoxu Zhao, ${ }^{1}$ Cuibo Liu, ${ }^{1}$ Junliang Sun, ${ }^{2 *}$ Kian Ping Loh $^{1 *}$

${ }^{1}$ Department of Chemistry, National University of Singapore, 3 Science Drive 3, Singapore 117543, Singapore.

${ }^{2}$ College of Chemistry and Molecular Engineering, Beijing National Laboratory for Molecular Sciences, Peking University, Beijing 100871, China.

${ }^{3}$ Department of Materials and Environmental Chemistry Stockholm University, SE-10691 Stockholm (Sweden).

${ }^{4}$ State Key Laboratory of Applied Organic Chemistry, College of Chemistry and Chemical Engineering, Lanzhou University, Lanzhou, Gansu 730000, China.

${ }^{5}$ School of Physical and Mathematical Sciences, Nanyang Technological University, 21 Nanyang Link, Singapore 637371.

${ }^{6}$ School of Physical Science and Technology, Shanghai Tech University, Shanghai 201210, China.

$\dagger$ These authors contributed equally to this work.

*Correspondence to: junliang.sun@ pku.edu.cn (J.S.); chmlohkp@nus.edu.sg (K.P.L.)

Abstract: Although polymers have been studied for well over a century, there are few examples of covalently linked polymer crystals synthesized directly from solution. One-dimensional (1D) covalent polymers that are packed into a framework structure can be viewed as a 1D covalent organic framework (COF), but making single crystal of this has been elusive. Herein, by combining labile metal coordination and dynamic covalent chemistry, we discovered a strategy to synthesize single-crystal metallo-COF under solvothermal conditions. The single-crystal structure was rigorously solved using single-crystal electron diffraction (SCED) technique. The noncentrosymmetric metallo-COF allows second harmonic generation (SHG). Due to the presence of syntactic pendant amine groups along the polymer chains, the metallopolymer crystal can be further cross-linked into a crystalline woven network.

Main Text: Framework solids have been one of the hottest materials over the past 30 years because they represent mankind's attempt to control chemical bonding in space versus random polymerisation $^{1,2}$. Depending on the nature of the strongest bond (coordination bond, covalent bond, hydrogen bond, etc.) used in constructing the solids, framework materials are categorized into metal-organic frameworks $(\mathrm{MOFs})^{3-5}$, covalent organic frameworks $(\mathrm{COFs})^{6-10}$, and hydrogen-bonded organic frameworks $(\mathrm{HOFs})^{11,12}$. The synthesis of framework materials needs to 
be carried out under conditions where bond formation is highly reversible to facilitate the selfcorrection process necessary for crystal growth. In this regard, COFs are among the most difficult to crystallize, owing to the lesser reversibility of their covalent linkages compared to coordination bonds and hydrogen bonds in MOFs and HOFs. The ease of encoding functionalities in COFs and their structural robustness render them potentially useful in wide-ranging applications ${ }^{13-19}$. However, an in depth understanding of the structure-property correlation in COFs is lacking, owing to fact that most synthesized COFs are polycrystalline, which hampers structural determination. Recently, the addition of crystal seeds or modulators have been used to grow singlecrystalline two- or three-dimensional (2D or 3D) $\mathrm{COFs}^{20,21}$. Nevertheless, only a few examples of $3 \mathrm{D}$ COFs have their crystal structure rigorously solved ${ }^{21-23}$. It is generally recognized that single crystalline frameworks of lower dimensionality are more difficult to grow compared to higher dimensionality ones ${ }^{24}$.

Although the vast choices of COF building units and covalent linkages give rise to diverse structural motifs in the synthesized product, the produced 2D and 3D COFs tend to crystallize in high symmetric space groups due to the high symmetries of the building blocks and low freedom of intermolecular packing, 8, As such, conventionally synthesized COFs are mostly centrosymmetric and do not exhibit second harmonic generation or ferroelectricity ${ }^{25}$. In this regard, one dimensional (1D) COFs, which possess a high degree of freedom in molecular packing, can be a candidate to construct non-centrosymmetric crystals. Conceptually, the framework structure in 1D COF is constructed from 1D-confined covalent linkages, and non-covalent interactions (such as $\pi-\pi$ interactions, hydrogen bonding, etc.) in the other two dimensions help to pack the 1D chains. However, the extremely high anisotropy and the entropy-driven random packing of organic chains impose a huge challenge on the synthesis of 1D COFs. Using its covalent analogue, linear polymers, as examples: since the invention of the first synthetic polymer (Bakelite) in 1907, nearly all the polymers synthesized are amorphous or poorly crystalline. To obtain a well-defined 1D COF, two basic aspects have to be considered: (i) How to get a framework, not just a densely packed organic polymer; (ii) how to control the periodic packing of organic chains to get a single crystalline structure. Although topochemical polymerisation approach has been used to produce single crystalline polymers from pre-packed molecular crystals, the approach is rather limited in scope $^{26-28}$. It is highly desirable to search for a direct crystallization method from solution, which has been so far elusive.

Herein, we demonstrate a strategy to synthesize single crystalline 1D metallo-COFs by combining metal-ligand coordination ${ }^{29}$ and dynamic covalent chemistry (DCC) ${ }^{30}$ (Fig. 1). A onepot reaction combining self-assembly ${ }^{31,32}$ and imine condensation was conducted under 35 solvothermal conditions. Along with the poly-condensation reaction, the constituents are consumed and regenerated from the reactant "pool"33. An advantage of this method is that building blocks can evolve into multi-intermediates through reversible reactions or interactions without the need to synthesize each building block individually, which is distinct from the conventional methods of making crystalline organic networks using predetermined building blocks ${ }^{34,35}$. We first attempt to construct 1D conjugated polymer solely based on DCC without a metal template. Due 
to the absence of secondary interactions, the 1D polymer chains pack randomly to afford a poorly crystalline polymer (Supplementary Fig. 1). We then introduce $\mathrm{AgBF}_{4}{ }^{36}$ to initiate ligand exchange and provide an additional reversible process for the poly-condensation, which should be beneficial for the crystal nucleation process. When one of the building units, 4,4'-(1,10-phenanthroline-2,9diyl)dianiline (I), is in excessive amount, it can be anchored onto the backbone chains via $\mathrm{Ag}^{\mathrm{I}}$ coordination in a syntactic fashion, thus providing periodic spacers to induce $\pi-\pi$ stacking and hydrogen bonding between the 1D chains (Fig.1). Using this approach, we synthesized single crystalline 1D metallo-COF (mCOF-Ag) with micrometer-sized particles, allowing the rigorous characterisation of the crystal structure via single-crystal electron diffraction (SCED) ${ }^{37}$.

In line with the strategy, a one-pot reaction by combining self-assembly and imine condensation was conducted under solvothermal conditions. In 2,9-bis(4(dimethoxymethyl)phenyl)-1,10-phenanthroline (II), an acetal group ${ }^{38}$ is preferred for its good solubility which is beneficial for the self-assembly process; besides, the relatively low reactivity of acetal with amine groups can reduce the crystal nucleation rate to enable good crystals. Once $\mathrm{AgBF}_{4}$ was added into the suspension of $\mathbf{I}$ and $\mathbf{I I}$, an immediate colour change was observed indicating the occurrence of self-assembly via coordination. The amount of $\mathrm{AgBF}_{4}$ added has a strong influence on crystal qualities. According to the strategy, the stoichiometry of I, II, and $\mathrm{AgBF}_{4}$ is 3:1:2. However, adopting this ratio produces a large number of $\mathrm{Ag}$ particles on the material. The presence of $\mathrm{Ag}$ particles is attributed to the reversible nature of imine bond; in situ 20 formed aldehyde groups can reduce $\mathrm{Ag}$ ions into elemental $\mathrm{Ag}$, which then aggregated into particles. When the ratio of $\mathrm{AgBF}_{4}$ is reduced to 1, no Ag particles were detected under the same reaction conditions. The use of excess I and II can protect Ag ions from being reduced. After carefully screening the reaction conditions, mCOF-Ag was obtained in a mixture of 1-butanol, 1,2dichlorobenzene, and $6 \mathrm{M}$ aqueous acetic acid $(1 / 9 / 1, \mathrm{v} / \mathrm{v} / \mathrm{v})$ at $120^{\circ} \mathrm{C}$ for three days with a feeding ratio of I, II, and $\mathrm{AgBF}_{4}$ equals to $3: 1: 1$. After copiously washing with DMSO and THF, and drying at $100{ }^{\circ} \mathrm{C}$ for 8 hours, mCOF-Ag was obtained as a light yellow solid (74\% yield, based on $\left.\mathrm{AgBF}_{4}\right)$.

The chemical bonding in mCOF-Ag was assessed by Fourier-transform infrared spectroscopy (FT-IR) (Supplementary Figs. 3 and 4 ) and ${ }^{13} \mathrm{C}$ cross-polarization magic-angle spinning (CP/MAS) NMR (Supplementary Fig. 5). mCOF-Ag exhibits sharp powder X-ray diffraction (PXRD) peaks, indicating its high crystallinity (Supplementary Fig. 2). Scanning electron microscopy (SEM) image reveals that mCOF-Ag exhibits a uniform rod-like morphology with the crystal size larger than $2 \mu \mathrm{m}$ (Fig. 2b). Ordered lattice fringes in the high-resolution transmission electron microscopy (HRTEM) image (Fig. 2c) and selected-area electron diffraction (SAED) pattern (Fig. 2c, inset) confirm its single-crystal nature.

The single-crystal structure of mCOF-Ag is determined directly from the SCED data ${ }^{39,40}$ (Fig. 2a). From the observed electron density maps (Fig. 2d and Supplementary Fig. 8), all positions of the non-hydrogen atoms $(\mathrm{C}, \mathrm{N}$, and $\mathrm{Ag})$ on the framework structure and the locations of the guests $\left(\mathrm{BF}_{4}^{-}\right.$anions) are identified. The SCED data was collected on a typical rod-like 
crystal, with a resolution of $\sim 0.95 \AA$, which reflects the single-crystal nature of mCOF-Ag. A $C$ centered monoclinic cell with $a=15.66 \AA, b=31.00 \AA, c=10.87 \AA$, and $\beta=123.31^{\circ}$ is identified by indexing the SCED data using the program XDS (Supplementary Table S1). As indicated by the reflection conditions ( $h k l: h+k ; 0 k l: k=2 n ; h k 0: h+k ; h 00: h=2 n$; and $0 k 0: k=2 n$ ), the possible space groups are deduced to be $C 2 / c$ (No.15), $C c$ (No. 9), $C 2 / m$ (No. 12), $C m$ (No. 8), or $C 2$ (No. 5). Based on this SCED data, the structure model of mCOF-Ag was solved directly in the space group $C 2 / c$ using the program SHELXT (dual-space method). In the determined structure model, it is found that the $\mathrm{C}$ and $\mathrm{N}$ atoms on the connection bonds $(-\mathrm{C}=\mathrm{N}-)$ are symmetry related. In order to make them symmetry independent, the symmetry of the structure model was reduced into its subgroup $C 2$. The determined structure model of mCOF-Ag was further confirmed and refined against its synchrotron powder X-ray diffraction (SPXD) data (Supplementary Fig. 11). Using the SVD-index method implemented in the software Topas, the SPXD data of mCOF-Ag is indexed by a $C$-centered monoclinic cell of $a=15.83 \AA, b=29.97 \AA, c=10.69 \AA$, and $\beta=123.96^{\circ}$, which is consistent with the unit cell obtained from the SCED data. The final agreement residuals for the Rietveld refinement are $R_{\mathrm{I}}=0.075, R_{\mathrm{wp}}=0.108$, with $R_{\exp }=0.049$.

Further analysis of the crystal structure reveals more insights about the packing of the 1D chains. The adjacent phenanthroline chains are arranged in a parallel fashion to generate a 2D corrugated layer (Supplementary Fig. 10), and the 2D layers are further stabilized by the interactions of amines with $\mathrm{BF}_{4}{ }^{-}$anions (Supplementary Fig. 9), as well as the $\pi-\pi$ stacking of interlayer phenanthroline rings (Supplementary Fig. 10), to form a permanent structure (Fig. 3a). The Ag and pendant groups $\mathbf{I}$ are arranged in a syntactic version, favouring ordered packing of the polymer chains. mCOF-Ag belongs to the $C 2$ space group, which is non-centrosymmetric. This is validated by second harmonic generation $(\mathrm{SHG})^{41}$ at $425 \mathrm{~nm}$ when the crystal is excited by fundamental laser wavelength of $850 \mathrm{~nm}$ (Fig. 3b and Supplementary Fig. 16). Polarizationdependent SHG was recorded on an isolated crystal. Using polarized laser excitation, a SHG map of the COF-Ag crystal could be obtained (Fig. 3b, inset). The strongest SHG response was observed under parallel polarized excitation, this means that the maximum SHG response originates from the longitudinal direction of the mCOF-Ag crystal.

Due to the packing of zigzag chains, the orientation and rotation of amine groups in building block $\mathbf{I}$ are spatially well defined and the two diagonally positioned $-\mathrm{NH}_{2}$ groups are separated by $\sim 3.9 \AA$ (Fig. 4a). mCOF-Ag is an ideal scaffold for crystalline state polymerisation because the adjacent amine groups can be linked by bi-functional compounds. Glyoxal, a molecule with two aldehyde groups, was chosen for its suitable molecular size and good reactivity with amine groups. mCOF-Ag was reacted with glyoxal solution $\left(40\right.$ wt. $\%$ in $\left.\mathrm{H}_{2} \mathrm{O}\right)$ at $70{ }^{\circ} \mathrm{C}$ in $1,4-$ dioxane. After incubation for five days and washing, the product was collected and dried to yield a cross-linked woven network, termed as wCOF-Ag (Fig. 4a). The crystalline-state polymerisation, which occurs via "stitching" of the inter-chain amine groups by the aldehyde molecules, was verified using a range of techniques. In the FT-IR spectra, the characteristic bands at $3361 \mathrm{~cm}^{-1}$, $3301 \mathrm{~cm}^{-1}$, and $3197 \mathrm{~cm}^{-1}$ for $\mathrm{N}-\mathrm{H}$ stretching, and at $1618 \mathrm{~cm}^{-1}$ for N-H bending, have almost vanished after cross-linking, indicating the near-complete consumption of amine groups in mCOF- 
Ag (Supplementary Fig. 17). The polymerisation process was further verified by the CP/MAS NMR spectrum of wCOF-Ag, where the peak corresponding to the aldehyde carbon in glyoxal at $190 \mathrm{ppm}$ is absent (Supplementary Fig. 18), indicating the full connection of amine and aldehyde groups. The PXRD pattern appears very similar with mCOF-Ag except for slight differences at high angles (Fig. 4b). These results affirm that cross-linking process did not affect the major part of the crystal structure. One evidence that the cross-linked solid is different from the pristine structure is provided by the photoluminescence (PL) spectroscopy, where the PL of wCOF-Ag showed a dramatic six-fold enhancement in intensity compared to that of mCOF-Ag (Supplementary Fig. 23). We have also examined the mechanical properties of the crystals before and after cross-linking using nano-indentation. By indenting on an isolated micron-sized crystal of each of these two samples, the Young's moduli of mCOF-Ag and wCOF-Ag were determined to be 9.0 (Supplementary Fig. 24) and 19.1 GPa (Supplementary Fig. 25), respectively (Fig. 4c). The value of wCOF-Ag is comparable with that of COF-505 $(\sim 12.5 \mathrm{GPa})^{34}$. The distinct increase in Young's modulus of wCOF-Ag is in line with its covalently connected structure in a 3D space, which imbues greater rigidity on the resulting material.

In this study, by combining metal-ligand coordination and dynamic imine bond formation, a single crystalline material, $\mathrm{mCOF}-\mathrm{Ag}$, was successfully constructed via solvothermal conditions; its crystal structure was rigorously solved with SCED. Due to its non-centrosymmetric structure, mCOF-Ag shows an obvious SHG signal, demonstrating its potential as nonlinear optical 20 materials. Moreover, due to the presence of interlaced pendant amine groups along the polymer chains, a high degree of control on the polymer backbone is obtained by crystalline-state polymerisation to form a woven network. From a synthetic perspective, the strategy we developed combines the powerful templating effect of metal ions and cooperative organization of building blocks to maximize the number of secondary bonds in solution, thus it can be a promising method for the solution-phase synthesis of single-crystal covalent metallopolymers with unique topologies and functionalities. 


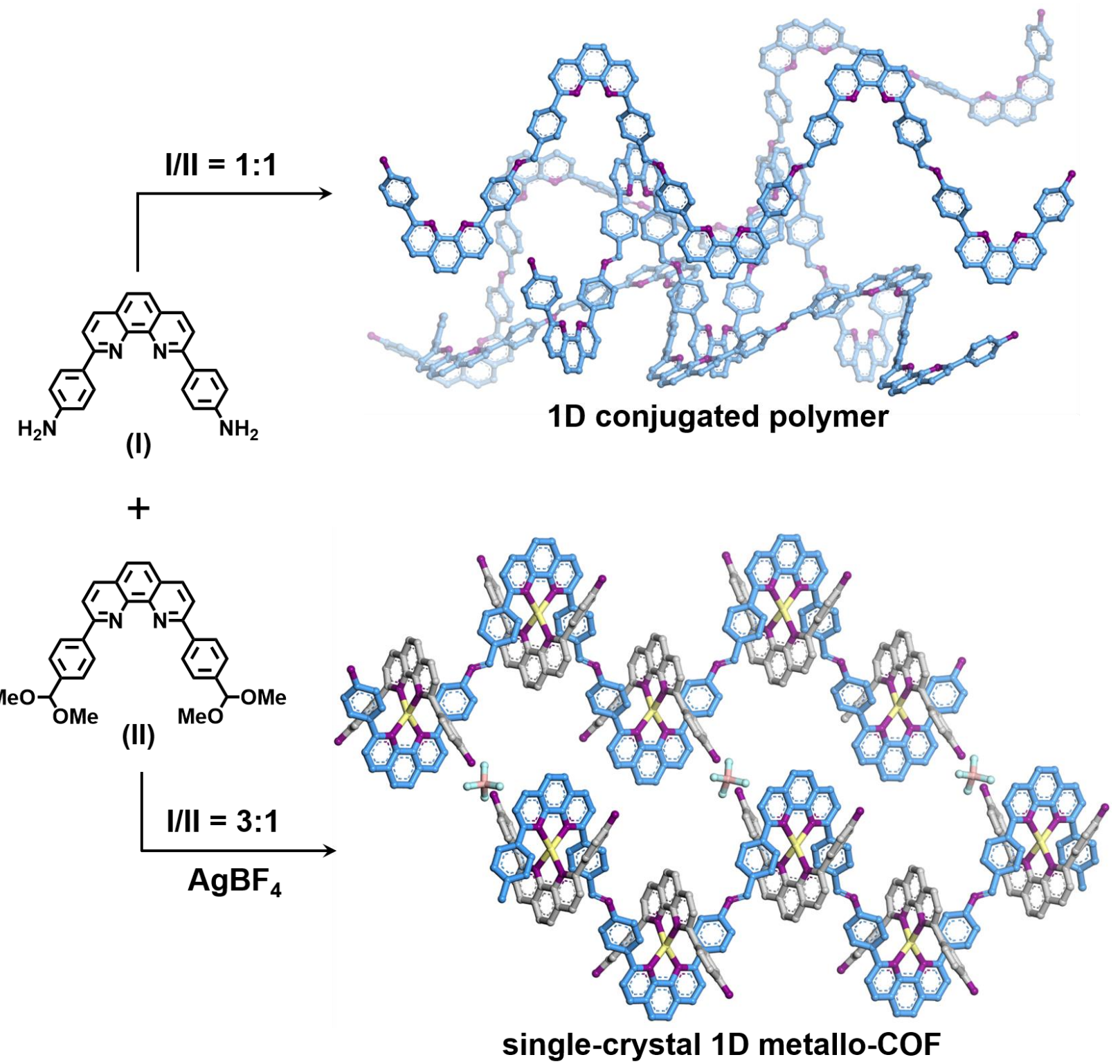

Fig. 1 | Strategy of synthesizing single crystalline $1 \mathrm{D}$ metallo-COF. Colour scheme: $\mathrm{C}$ on organic chains, blue; $\mathrm{C}$ on pendants, grey; N, purple; Ag, yellow; B, pink; F, green. Hydrogen atoms are omitted for clarity. 

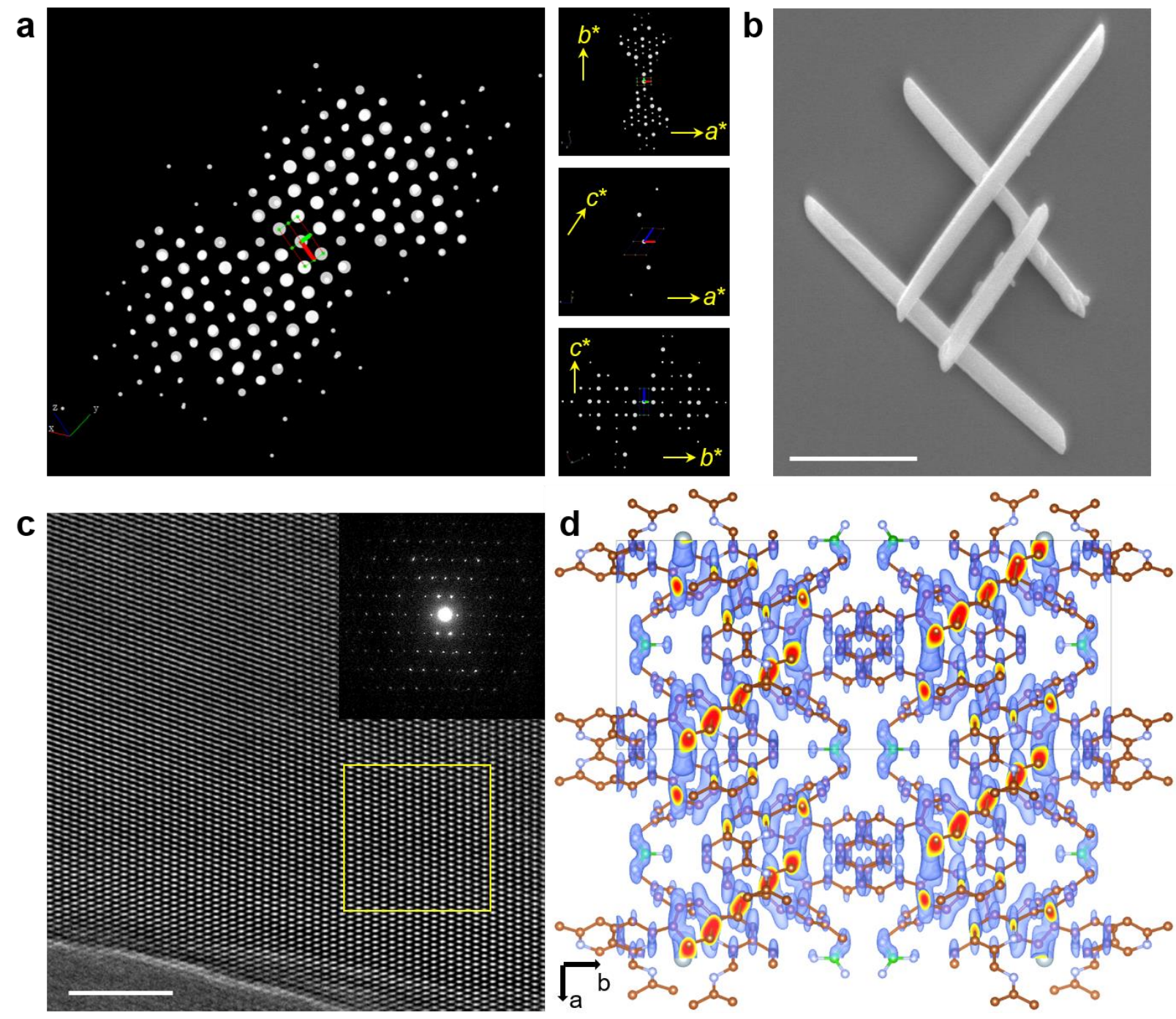

Fig. 2 Structure characterisation and analysis of $\mathbf{m C O F}-\mathbf{A g}$. a, 3D reciprocal lattice of mCOF$\mathrm{Ag}$ reconstructed from the SCED data (left) and 2D slices cut from the reconstructed reciprocal lattice (right). b, SEM image of mCOF-Ag with uniform rod-like morphology. Scale bar: $1 \mu \mathrm{m}$. c, HRTEM image of mCOF-Ag and SAED pattern (inset) confirmed the single crystalline nature of the material. Scale bar: $20 \mathrm{~nm}$. d, Observed electron density map of the initial structure model determined from SCED data along the $c$-axis. 

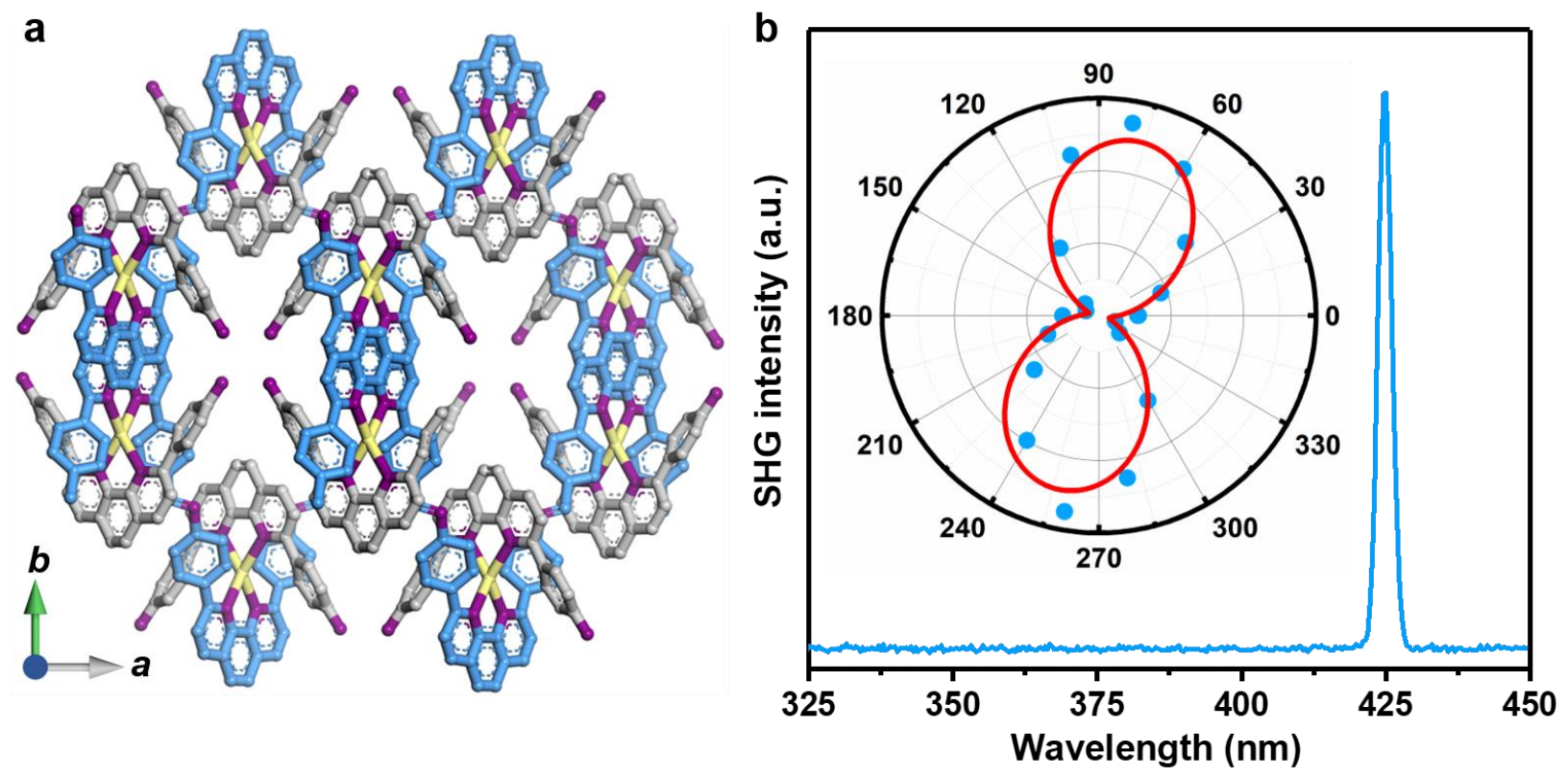

Fig. 3 | Single-crystal structure and nonlinear optical measurements of mCOF-Ag. a, Crystal view of mCOF-Ag along the $c$-axis. Colour scheme: $\mathrm{C}$ on organic chains, blue; $\mathrm{C}$ on pendants, grey; $\mathrm{N}$, purple; $\mathrm{Ag}$, yellow. Hydrogen atoms and $\mathrm{BF}_{4}{ }^{-}$anions are omitted for clarity. $\mathbf{b}$, The SHG spectrum of an isolated crystal of mCOF-Ag. The excitation wavelength is $850 \mathrm{~nm}$, the peak at $425 \mathrm{~nm}$ is the SHG signal. Inset: polarization dependent SHG response. Red curve is the fitting result of experimental data (blue dots). The units are in degree. 


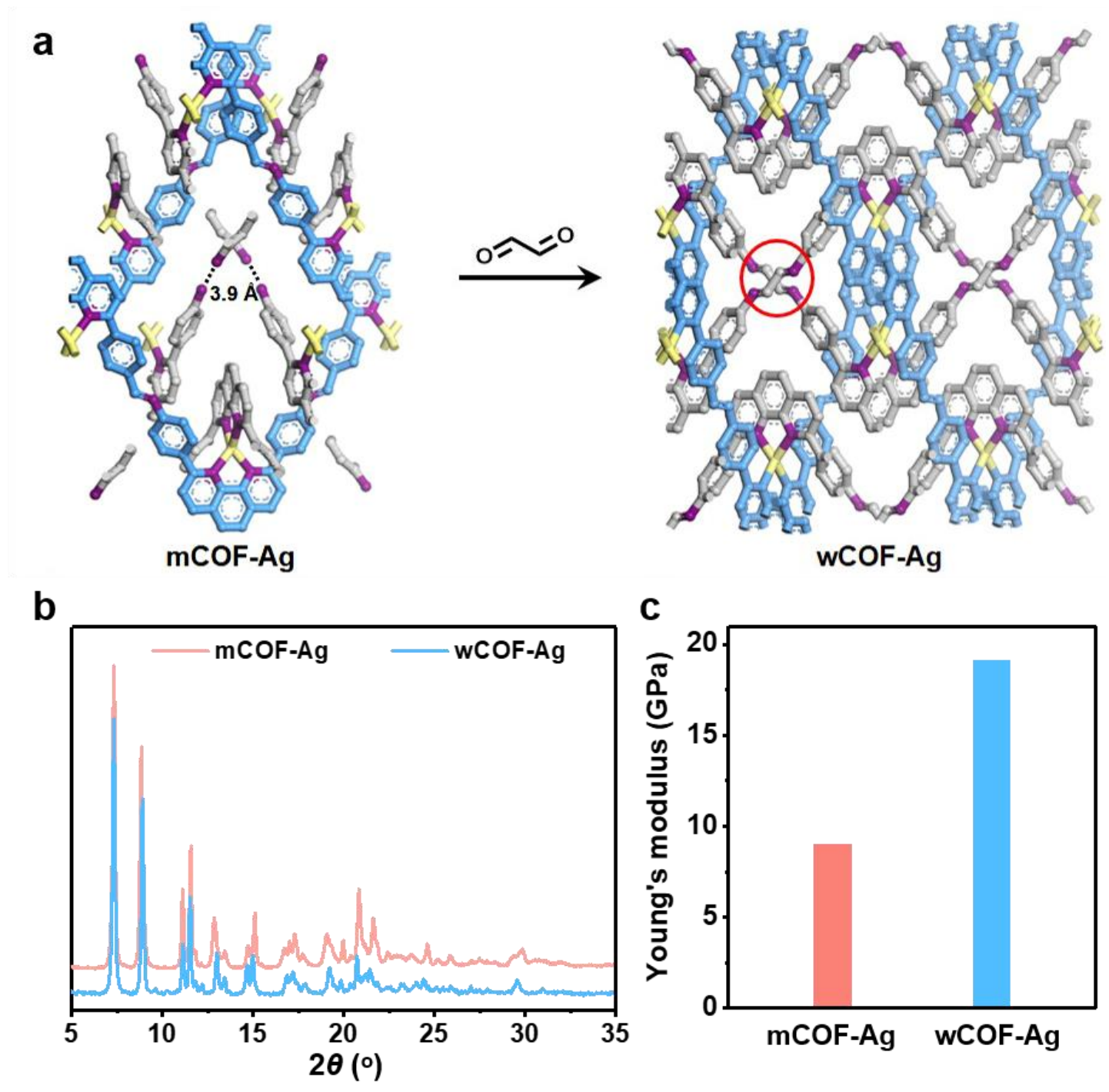

Fig. 4 | Crystalline-state polymerisation and characterisation. a, Confined environments of mCOF-Ag (left) and the ideal structure of the cross-linked framework, wCOF-Ag (right). One of the cross-linked parts is highlighted with a red circle. Colour scheme: $\mathrm{C}$ on organic chains, blue; $\mathrm{C}$ on pendants, grey; $\mathrm{N}$, purple; $\mathrm{Ag}$, yellow. Hydrogen atoms and $\mathrm{BF}_{4}{ }^{-}$anions are omitted for clarity. b, PXRD patterns of mCOF-Ag and wCOF-Ag. $\mathbf{c}$, The Young's moduli of mCOF-Ag and wCOF-Ag. 


\section{References:}

1. Yaghi, O. M., O'Keeffe, M. \& Kanatzidis, M. Design of solids from molecular building blocks: Golden opportunities for solid state chemistry. J. Solid State Chem. 152, 1-2 (2000).

2. Yaghi, O. M. et al. Reticular synthesis and the design of new materials. Nature 423, 705-714 (2003).

3. Zhou, H.-C., Long, J. R. \& Yaghi, O. M. Introduction to metal-organic frameworks. Chem. Rev. 112, 673-674 (2012).

4. Kitagawa, S., Kitaura, R. \& Noro, S.-i. Functional porous coordination polymers. Angew. Chem. Int. Ed. 43, 2334-2375 (2004).

10 5. Furukawa, H., Cordova, K. E., O’Keeffe, M. \& Yaghi, O. M. The chemistry and applications of metal-organic frameworks. Science 341, 1230444 (2013).

6. Cote, A. P. et al. Porous, crystalline, covalent organic frameworks. Science 310, 1166-1170 (2005).

7. Diercks, C. S. \& Yaghi, O. M. The atom, the molecule, and the covalent organic framework.

$15 \quad$ Science 355, eaal1585 (2017).

8. Huang, N., Wang, P. \& Jiang, D. Covalent organic frameworks: A materials platform for structural and functional designs. Nat. Rev. Mater. 1, 16068 (2016).

9. Ding, S.-Y. \& Wang, W. Covalent organic frameworks (COFs): From design to applications. Chem. Soc. Rev. 42, 548-568 (2013).

20 10. Segura, J. L., Mancheño, M. J. \& Zamora, F. Covalent organic frameworks based on schiffbase chemistry: Synthesis, properties and potential applications. Chem. Soc. Rev. 45, 5635-5671 (2016).

11. Brunet, P., Simard, M. \& Wuest, J. D. Molecular tectonics. Porous hydrogen-bonded networks with unprecedented structural integrity. J. Am. Chem. Soc. 119, 2737-2738 (1997).

12. He, Y., Xiang, S. \& Chen, B. A microporous hydrogen-bonded organic framework for highly selective $\mathrm{C}_{2} \mathrm{H}_{2} / \mathrm{C}_{2} \mathrm{H}_{4}$ separation at ambient temperature. J. Am. Chem. Soc. 133, 14570-14573 (2011).

13. Lin, S. et al. Covalent organic frameworks comprising cobalt porphyrins for catalytic $\mathrm{CO}_{2}$ reduction in water. Science 349, 1208-1213 (2015).

14. Xu, H., Gao, J. \& Jiang, D. Stable, crystalline, porous, covalent organic frameworks as a platform for chiral organocatalysts. Nat. Chem. 7, 905-912 (2015).

15. DeBlase, C. R. et al. $\beta$-ketoenamine-linked covalent organic frameworks capable of pseudocapacitive energy storage. J. Am. Chem. Soc. 135, 16821-16824 (2013).

16. Li, X. et al. Covalent-organic-framework-based Li- $\mathrm{CO}_{2}$ batteries. Adv. Mater. 0, 1905879 (2019).

17. Guan, X. et al. Chemically stable polyarylether-based covalent organic frameworks. Nat. Chem. 11, 587-594 (2019).

18. Li, X. et al. Tuneable near white-emissive two-dimensional covalent organic frameworks. Nat. Commun. 9, 2335 (2018). 
19. Dogru, M. et al. A photoconductive thienothiophene-based covalent organic framework showing charge transfer towards included fullerene. Angew. Chem. Int. Ed. 52, 2920-2924 (2013). 20. Evans, A. M. et al. Seeded growth of single-crystal two-dimensional covalent organic frameworks. Science 361, 52-57 (2018).

21. Ma, T. et al. Single-crystal x-ray diffraction structures of covalent organic frameworks. Science 361, 48-52 (2018).

22. Beaudoin, D., Maris, T. \& Wuest, J. D. Constructing monocrystalline covalent organic networks by polymerization. Nat. Chem. 5, 830 (2013).

23. Sun, T. et al. Atomic-level characterization of dynamics of a 3D covalent organic framework by cryo-electron diffraction tomography. J. Am. Chem. Soc. 141, 10962-10966 (2019).

24. Navarro, J. A. R. The dynamic art of growing COF crystals. Science 361, 35-35 (2018).

25. Ok, K. M., Chi, E. O. \& Halasyamani, P. S. Bulk characterization methods for noncentrosymmetric materials: Second-harmonic generation, piezoelectricity, pyroelectricity, and ferroelectricity. Chem. Soc. Rev. 35, 710-717 (2006).

26. Dou, L. et al. Single-crystal linear polymers through visible light-triggered topochemical quantitative polymerization. Science 343, 272-277 (2014).

27. Lauher, J. W., Fowler, F. W. \& Goroff, N. S. Single-crystal-to-single-crystal topochemical polymerizations by design. Acc. Chem. Res. 41, 1215-1229 (2008).

28. Kissel, P. et al. A two-dimensional polymer prepared by organic synthesis. Nat. Chem. 4, 287$20291(2012)$.

29. Lewis, J. E. M., Beer, P. D., Loeb, S. J. \& Goldup, S. M. Metal ions in the synthesis of interlocked molecules and materials. Chem. Soc. Rev. 46, 2577-2591 (2017).

30. Rowan, S. J. et al. Dynamic covalent chemistry. Angew. Chem. Int. Ed. 41, 898-952 (2002).

31. Gil-Ramírez, G., Leigh, D. A. \& Stephens, A. J. Catenanes: Fifty years of molecular links. Angew. Chem. Int. Ed. 54, 6110-6150 (2015).

32. Nitschke, J. R. Construction, substitution, and sorting of metallo-organic structures via subcomponent self-assembly. Acc. Chem. Res. 40, 103-112 (2007).

33. Corbett, P. T. et al. Dynamic combinatorial chemistry. Chem. Rev. 106, 3652-3711 (2006).

34. Liu, Y. et al. Weaving of organic threads into a crystalline covalent organic framework. Science $30 \quad 351,365-369(2016)$.

35. Liu, Y. et al. 3D covalent organic frameworks of interlocking 1D square ribbons. J. Am. Chem. Soc. 141, 677-683 (2019).

36. Khlobystov, A. N. et al. Supramolecular design of one-dimensional coordination polymers based on silver(I) complexes of aromatic nitrogen-donor ligands. Coord. Chem. Rev. 222, 155-192 $35 \quad(2001)$.

37. Wang, Y. et al. Elucidation of the elusive structure and formula of the active pharmaceutical ingredient bismuth subgallate by continuous rotation electron diffraction. Chem. Commun. 53, 7018-7021 (2017).

38. Li, Z.-J. et al. Synthesis of $-\mathrm{C}=\mathrm{N}$ - linked covalent organic frameworks via the direct condensation of acetals and amines. Chem. Commun. 52, 7217-7220 (2016). 
39. Ma, T. et al. Observation of interpenetration isomerism in covalent organic frameworks. J. Am. Chem. Soc. 140, 6763-6766 (2018).

40. Gao, C. et al. Isostructural three-dimensional covalent organic frameworks. Angew. Chem. Int. Ed. 58, 9770-9775 (2019).

41. EATON, D. F. Nonlinear optical materials. Science 253, 281-287 (1991).

\section{Acknowledgments}

K.P.L. acknowledges NRF-CRP grant "Two Dimensional Covalent Organic Framework: Synthesis and Applications". Grant number NRF-CRP16-2015-02, funded by National Research Foundation, Prime Minister's Office, Singapore. J.S. and Y.L. acknowledge National Natural Science Foundation of China and Swedish Research Council. H.-S.X. and K.P.L. thank Q. Zhang (National University of Singapore) for help with PL tests, Z. Zhu, X. Wu, Q. Guo (National University of Singapore) for help with SHG tests, and L. Zhang, P. Chen (Peking University) for help with single-crystal structure analysis.

\section{Author contributions}

15 K.P.L. supervised the project. J.S. supervised the crystal structures analysis. H.-S.X. designed and performed the experiments. Y.L. conducted SCED characterisation and Rietveld Refinement. X.L., Z.C., T.M., L.L., K.L., X.F.L. and C.L. discussed the synthesis and characterisation. I.A. conducted the SHG test. L.W. conducted the AFM test. R.L. helped to analyze the nanoindentation data. X.S. conducted CP/MAS NMR characterisation. Y.Z. conducted HRTEM characterisation. X.Z. helped to analyse the HRTEM data. K.P.L., H.-S.X., X.L., P.Z.S. and Y.L. wrote the manuscript.

\section{Competing interests}

The authors declare no competing interests. 